Journal of The Faculty of Science and Technology (JFST)

http://journal.oiu.edu.sd/index.php/JFST

https://doi.org/10.52981/jfst.vi7.948

ISSN: 1858 - 6007

مجلة العلوم و التقانة ـ جامعة أم درمان الإسلامية ـ وزارة التعليم العالي و البحث العلمي ـ السودان

Research article

JFST Issue No. 7 (2020) 19- 32

\title{
Group Classification Invariant Solutions of Burgers' Equation
}

\section{Mohammed Adam Abdualah Khatir'1, Mohammed Ali Basher², Blegiss \\ Abdulaziz Abdulrahman Ebyed ${ }^{3}$}

${ }^{1,3}$ Sudan University of Sciences and Technology, AL-Neelain University ${ }^{2}$

\section{E. Mail: mohamedkhatir68@gmail.com}

\begin{abstract}
:
The aims of the present paper is to solve the problem of the group classification of the general Burgers' equation $u_{t}=f(x, u) u_{x}^{2}+g(x, u) u_{x x}$, where $f$ and $g$ are arbitrary smooth functions of the variables $x$ and $u$, by using Lie method. The paper is one of the few applications of an algebraic approach to the problem of group classification: We followed the analysis mathematical method using the method of preliminary group classification. A number of new interesting nonlinear invariant models which have nontrivial invariance algebras are obtained. The result of the work is a wide class of equations summarized in table form.
\end{abstract}

Key words: Infinitesimal generator, Symmetries of Burgers' equation, optimal system, group classification of algebra.

\section{Introduction:}

It is well known that the symmetry group method plays an important role in the analysis of differential equations. The history of group classification methods goes back to Sophus Lie. The first paper on this subject is S.Lie, Arech(1881), where Lie proves that a linear two-dimensional second-order PDE may admit at most a threeparameter invariance group (apart from the trivial infinite parameter symmetry group, which is due to linearity). He computed the maximal invariance group of the onedimensional heat conductivity equation and utilized this symmetry to construct its explicit solutions. Saying it the modern way, he performed symmetry reduction of the 
heat equation. Nowadays symmetry reduction is one of the most powerful tools for solving nonlinear partial differential equations (PDEs). Recently, there have been several generalizations of the classical Lie group method for symmetry reductions. L.V.Ovsiannikov (1982), developed the method of partially invariant solutions. This approach is based on the concept of an equivalence group. Which is a Lie transformation group acting in the extended space of independent variables, functions and their derivatives. And preserving the class of partial differential equations. The investigation of the exact solutions plays an important role in the study of nonlinear physical systems. A wealth of methods have been developed to find those exact physically significant solutions of a PDE though it is rather difficult. Some of the most important methods are the inverse scattering method R.M.Miura (1967), Darboux and Böcklund transformations Y.S.Li. (1999), Hirota bilinear method R.Hirota.J.Satsuma(1976) Lie symmetry analysis B.J. Cantwell (2002), etc. the paper H.L.J.Liu.J Zhangb(2008), based on the Lie group method. Is investigated a very famous an important equation, which is the general Burgers' equation as the form

$$
u_{t}=a u_{x}^{2}+b u_{x x}
$$

where $u=u(x, t)$ is the unknown real function. $a, b \in R$ and $a b \neq 0$. In the present paper. We consider the general Burgers' equation as the form

$$
u_{t}=f(x, u) u_{x}^{2}+g(x, u) u_{x x}
$$

Where $u=u(x, t)$ is the unknown real function, $f$ and $g$ are arbitrary smooth functions of the variables $x$ and $u$. Eq. (1.1.2) represents the Burgers' equation combining both dissipative and nonlinear effects, therefore appears in a wide variety of physical applications. So it is important to lucubrate the exact explicit solutions and similarity reductions for this equation M.Nadjafikhah (2008). Here, we got the preliminary group classification of Eq. (1.1.2) by means of Lie point symmetry, and the constructed optimal systems of subalgebras. The knowledge of the optimal system of subalgebras gives the possibility of constructing the optimal system of solutions M.L.G,M.T.A.Valenti (2004) and permits the generation of new solutions starting form invariant or non-invariant solutions.

(1.1) Symmetries of Burgers' Equations: Let a partial differential equation contains $p$ dependent variables and $q$ independent variables. The one 
parameter Lie group of transformations $\tilde{x}=x_{i}+c \xi^{i}(x, u)+o\left(c^{2}\right): \quad \tilde{u}_{\alpha}=$ $u_{\alpha}+\epsilon \varphi^{\alpha}(x, u)+o\left(\epsilon^{2}\right)$,

Where $\xi^{i}=\left.\frac{\partial \tilde{x}_{i}}{\partial \epsilon}\right|_{\epsilon=0}, i=1, \ldots, p$, and $\varphi^{\alpha}=\left.\frac{\partial \widetilde{u}_{\alpha}}{\partial \epsilon}\right|_{\epsilon=0}, \alpha=1, \ldots, q$, are given.

The action of the Lie group can be recovered from that of its associated infinitesimal generators, we consider general vector field

$$
V=\sum_{i=1}^{p} \xi^{i}(x, u) \frac{\partial}{\partial x_{i}}+\sum_{\alpha=1}^{q} \varphi^{\alpha}(x, u) \frac{\partial}{\partial u^{\alpha}}
$$

On the space of independent and dependent variables. Therefore, the characteristic of the vector field $\mathrm{V}$ given by (1.2.4) is the function

$$
Q^{\alpha}\left(x,(u)^{(1)}\right)=\varphi^{\alpha}(x, u)-\sum_{i=1}^{p} \xi^{i}(x, u) \frac{\partial u^{\alpha}}{\partial x_{i}}, \quad \alpha=1, \ldots, q
$$

The second prolongation of infinitesimal operator

$$
X=\xi^{i}(x, t, u) \frac{\partial}{\partial x}+\xi^{2}(x, t, u) \frac{\partial}{\partial t} \varphi(x, t, u) \frac{\partial}{\partial u}
$$

Obtained via the following prolongation formulas:

$$
X^{(2)}=X+\varphi^{x} \frac{\partial}{\partial u_{x}}+\varphi^{t} \frac{\partial}{\partial u_{t}}+\varphi^{x t} \frac{\partial}{\partial u_{x x}}
$$

The coefficients are obtained by

$$
\varphi^{t}=D_{t} Q+\xi^{i} u_{x i}+\xi^{2} u_{t i}, \quad \varphi^{t J}=D_{t}\left(D_{J} Q\right)+\xi^{1} u_{x i J}+\xi^{2} u_{t i J}
$$

Where $Q=\varphi-\xi^{1} u_{x}-\xi^{2} u_{t}$ is the characteristic of the vector field $\mathrm{V}$ given by (1.2.4). For instance

$$
\begin{gathered}
\varphi^{x}=D_{x} \varphi-u_{x} D_{x} \xi^{1}-u_{t} D_{x} \xi^{2}, \\
\varphi^{t}=D_{t} \varphi-u_{x} D_{t} \xi^{1}-u_{t} D_{t} \xi^{2}, \\
\varphi^{x x}=D_{x} \varphi^{x}-u_{x x} D_{x} \xi^{1}-u_{x t} D_{x} \xi^{2}, \\
\varphi^{x t}=D_{x} \varphi^{x}-u_{x x} D_{t} \xi^{1}-u_{x t} D_{t} \xi^{2},
\end{gathered}
$$


where the operators $D_{x}$ and $D_{t}$ denotes the total derivatives with respect to $x$ and $t$ :

$$
\begin{gathered}
D_{x}=\frac{\partial}{\partial x}+u_{x} \frac{\partial}{\partial u}+u_{x x} \frac{\partial}{\partial u_{x}}+u_{x t} \frac{\partial}{\partial u_{t}}+\cdots \\
D_{t}=\frac{\partial}{\partial t}+u_{t} \frac{\partial}{\partial u}+u_{t t} \frac{\partial}{\partial u_{t}}+u_{t x} \frac{\partial}{\partial u_{x}}+\cdots
\end{gathered}
$$

by the theorems, in Y.S.Li. (1999). $\left.X^{(2)}\left[u_{t}-f(x, u) u_{x}^{2}-g(x, u) u_{x x}\right]\right|_{(1,2)}=0$. Since

$$
\begin{aligned}
& X^{(2)}\left[u_{t}-f(x, u) u_{x}^{2}-g(x, u) u_{x x}\right] \\
& =\varphi^{t}-\left(f_{x} \xi^{1}+f_{u} \varphi\right) u_{x}^{2}-\left(g_{x} \xi^{1}+g_{u} \varphi\right) u_{x x}-2 f \varphi^{x} u_{x}-g \varphi^{x x},
\end{aligned}
$$

therefore we obtain the following determining function:

$$
\begin{gathered}
{\left.\left[\varphi^{t}-\left(f_{x} \xi^{1}+f_{u} \varphi\right) u_{x}^{2}-\left(g_{x} \xi^{1}+g_{u} \varphi\right) u_{x x}-2 f \varphi^{x} u_{x}-g \varphi^{x x}\right]\right|_{(1,2)}} \\
=0
\end{gathered}
$$

In the case of arbitrary $f(x, u)$ and $g(x, u)$ it follows that

$$
\xi^{1}=\varphi=\varphi^{x}=\varphi^{t}=\varphi^{x x}=0,
$$

Or, $\xi^{1}=\varphi=0, \quad \xi^{2}=c$.

Therefore, for arbitrary $f(x, u)$ and $g(x, u)$ Eq. (1.1.1).admits the one-dimensional Lie algebra $g_{1}$, with the basis

$$
X_{2}=\frac{\partial}{\partial t}
$$

$g_{1}$ is called the principle Lie algebra for Eq. (1.1.1). So, it is remained to specify the coefficients $f$ and $g$ such that Eq. (1.1.1) admits an extension of the principal algebra $g_{1}$. Usually, the group classification is obtained by inspecting the determining equation. But in our case the complete solution of the determining equation (1.2.12) is a wasteful venture. Therefore, we don't solve the determining equation but, instead we obtain a partial group classification of Eq. (1.1.1) via the so-called method of preliminary group classification. This method was applied when an equivalence group is generated by a finite-dimensional Lie algebra $g_{s}$. The essential part of the method is the classification of all nonsimilar subalgebras of $g_{s}$. Actually, the application of the 
method is simple and effective when the classification is based on finite-dimensional equivalence algebra $g_{s}$.

(1.2) Equivalence Transformations: An equivalence transformation is a nondegenerate change of the variables $t, x, u$ taking any equation of the form (1.1.1) into an equation of the same form, generally speaking, with different $f(u, x)$ and $g(x, u)$. The set of all equivalence transformations forms an equivalence group s. we shall find a continuous subgroup $s_{C}$ of it making use of the infinitesimal method. We consider an operator of the group $s_{C}$ in the form

$$
\begin{aligned}
Y=\xi^{1}(x, t, u) & \frac{\partial}{\partial x}+\xi^{2}(x, t, u) \frac{y}{\partial t}+\varphi(x, t, u) \frac{\partial}{\partial u}+\mu(x, t, u, f, g) \frac{\partial}{\partial f} \\
& +v(x, t, u, f, g) \frac{\partial}{\partial g}
\end{aligned}
$$

from the invariance conditions of Eq. (1.1.1) written the system:

$$
\begin{aligned}
& u_{t}-f(x, u) u_{x}^{2}-g(x, u) u_{x x}=0 \\
& f_{t}=g_{t}=0
\end{aligned}
$$

where $u, f$ and $g$ are considered as differential variables: $u$ on the space $(x, t)$ and $f, g$ on the extended space $(x, t, u)$. The invariance conditions of the system (1.3.17) are

$$
\begin{aligned}
& Y^{(2)}\left(u_{t}-f(x, u) u_{x}^{2}-g(x, u) u_{x x}\right)=0 . \\
& Y^{(2)}\left(f_{t}\right)=Y^{(2)}\left(g_{t}\right)=0,
\end{aligned}
$$

where $Y^{(2)}$ is the prolongation of the operator (3.16):

$$
Y^{(2)}=Y+\varphi^{x} \frac{\partial}{\partial u_{x}}+\varphi^{t} \frac{\partial}{\partial u_{t}}+\varphi^{x t} \frac{\partial}{\partial u_{x t}} \varphi^{x x} \frac{\partial}{\partial u_{x x}}+\mu^{t} \frac{\partial}{\partial f_{t}}+v^{t} \frac{\partial}{\partial g_{t}}
$$

The coefficients $\varphi^{x}, \varphi^{t}, \varphi^{x t}, \varphi^{t t}$ are given in (1.2.7) and the other coefficients of (1.3.20) are obtained by applying the prolongation procedure to differential variables $f$ and $g$ with independent variables $(x, u)$. In view of (1.3.18), we have

$$
\mu^{t}=\widetilde{D}_{t}(\mu)-f_{x} \widetilde{D}_{t}\left(\xi^{1}\right)-f_{u} \widetilde{D}_{t}(\varphi), v^{t}=\widetilde{D}_{t}(v)-g_{x} \widetilde{D}_{t}\left(\xi^{1}\right)-g_{u} \widetilde{D}_{t}(\varphi)
$$

Where $\widetilde{D}_{t}=\frac{\partial}{\partial t}$. So, we have the following prolongation formulas: 


$$
\mu^{t}=\mu t-f_{x} \xi_{t}^{1}-f_{u} \varphi_{t}, \quad v^{t}=v t-g_{x} \xi_{t}^{1}-g_{u} \varphi_{t}
$$

The invariance conditions (3.19) give rise to

$$
\mu^{t}=v^{t}=0
$$

that is hold for every $f$ and $g$. We obtained

$$
\mu^{t}=v t=0 . \quad \xi_{t}^{1}=\varphi_{t}=0 .
$$

Moreover we obtained

$$
\varphi^{t}=2 f(x, u) u_{x} \varphi^{x}=g(x, u) \varphi^{x x}-\mu u_{x}^{2}-v u_{x x}-v=0 .
$$

The introducing the relation $u_{t}=f(x, u) u_{x}^{2}+g(x, u) u_{x x}$ to eliminate $u_{t}$ we are left with a polynomial equation involving the various derivatives of $u(x, t)$ whose coefficients are certain derivatives of $\xi^{1}, \xi^{2}, \varphi, \mu$, and $v$. We can equate the individual coefficients to zero, leading to the complete set of determining equations:

$$
\begin{aligned}
& \xi^{1}=\xi^{1}(x) \\
& \xi^{2}=\xi^{t}=0 \\
& \varphi_{t t}=\xi_{t}^{2} \\
& v=g \xi_{t}^{2}+2 \xi_{x}^{i} \\
& \mu=-f \xi_{t}^{2}-f\left(\varphi_{u}-2 \xi_{x}^{1}\right)-g \varphi_{u u} .
\end{aligned}
$$

So, we found that

$$
\begin{aligned}
& \xi^{1}(x)=a(x), \quad \xi^{2}=c_{1} t+c_{2}, \quad \varphi(x, u)=c_{1} u+b(x), \\
& \mu=-2 f\left(c_{1}-a(x)\right), \quad v=-g\left(c_{1}-a^{t}(x)\right),
\end{aligned}
$$

with constants $c_{1}, c_{2}$ and two arbitrary functions $a(x)$ and $b(x)$ such that

$b^{\prime \prime}(x)=c_{1}-a^{\prime}(x)$. We summarized: The class of Eq. (1.1.2) has an infinite continuous group of equivalence transformations generated by the following infinitesimal operators: 
$Y=a(x) \frac{\partial}{\partial x}+\left(c_{1} t+c_{2}\right) \frac{\partial}{\partial t}+\left(c_{1} u+b(x)\right) \frac{\partial}{\partial u}-2 f\left(c_{1}-a(x)\right) \frac{\partial}{\partial f}-\quad g\left(c_{1}-\right.$

$\left.\left.a^{\prime}\right)\right) \frac{\partial}{\partial g}$

therefore the symmetry algebra of the Burgers' equation (1.1.2) is spanned by the vector fields

$$
\begin{aligned}
& Y_{1}=t \frac{\partial}{\partial t}+u \frac{\partial}{\partial u}-2 f \frac{\partial}{\partial f}-g \frac{\partial}{\partial g}, \quad Y_{2}=\frac{\partial}{\partial t^{\prime}} \\
& Y_{3}=a(x) \frac{\partial}{\partial x}+2 f a(x) \frac{\partial}{\partial f}+g a^{\prime}(x) \frac{\partial}{\partial g}, \quad Y_{4}=b(x) \frac{\partial}{\partial u} .
\end{aligned}
$$

Table (a): Commutation relations satisfied by infinitesimal generators in (1.4.34)

\begin{tabular}{|c|c|c|c|c|c|}
\hline$[]$, & $Y_{1}$ & $Y_{2}$ & $Y_{3}$ & $Y_{4}$ & $Y_{5}$ \\
\hline$Y_{1}$ & $Y_{1}$ & 0 & 0 & 0 & 0 \\
$Y_{2}$ & $Y_{1}$ & 0 & 0 & $Y_{2}$ & 0 \\
$Y_{3}$ & $Y_{1}$ & 0 & 0 & $Y_{3}$ & 0 \\
$Y_{4}$ & $Y_{1}$ & $-Y_{2}$ & $-Y_{3}$ & 0 & 0 \\
$Y_{5}$ & $Y_{1}$ & 0 & 0 & 0 & 0 \\
\hline
\end{tabular}

Table (b): Adjoint relations satisfied by infinitesimal generators in (1.4.34)

\begin{tabular}{|c|c|c|c|c|c|}
\hline$[]$, & $Y_{1}$ & $Y_{2}$ & $Y_{3}$ & $Y_{4}$ & $Y_{5}$ \\
\hline$Y_{1}$ & $Y_{1}$ & $Y_{2}$ & $Y_{3}$ & $Y_{4}$ & $Y_{5}$ \\
$Y_{2}$ & $Y_{1}$ & $Y_{2}$ & $Y_{3}$ & $Y_{4}-s Y_{2}$ & $Y_{5}$ \\
$Y_{3}$ & $Y_{1}$ & $Y_{2}$ & $Y_{3}$ & $Y_{4}-s Y_{3}$ & $Y_{5}$ \\
$Y_{4}$ & $Y_{1}$ & $e^{\alpha} Y_{2}$ & $e^{\alpha} Y_{3}$ & $Y_{4}$ & $Y_{5}$ \\
$Y_{5}$ & $Y_{1}$ & $Y_{2}$ & $Y_{3}$ & $Y_{4}$ & $Y_{5}$ \\
\hline
\end{tabular}

Moreover, in the group of equivalence transformations there are included also discrete transformations, i.e. reflections

$$
t \rightarrow-t, \quad x \rightarrow-x, \quad u \rightarrow-u, \quad f \rightarrow-f, \quad g \rightarrow-g .
$$

\section{(1.4) Group Classification of Lie Algebras:}

One can observe in many applications of group analysis that most of extensions of the principal Lie algebra admitted by the equation under consideration are taken from the 
equivalence algebra $g_{s}$. We call these extensions s-extensions of the principal Lie algebra. The classification of all nonequivalent equations (with respect to a given equivalence group $G_{s}$.) admitting s-extensions of the principal Lie algebra is called a preliminary group classification. Here, $G_{S}$ is not necessary the largest equivalence group but, it can be any subgroup of the group of all equivalence transformations. So, we can take any finite-dimensional subalgebra (desirable as large as possible) of an infinite-dimensional algebra with basis (3.32) and use it for a preliminary group classification. We select the subalgebra $g_{5}$ spanned on the following operators:

$$
\begin{gathered}
Y_{1}=\frac{\partial}{\partial x}, \quad Y_{2}=\frac{\partial}{\partial t}, \quad Y_{3}=\frac{\partial}{\partial u} \\
Y_{4}=t \frac{\partial}{\partial t}+u \frac{\partial}{\partial u}-2 f \frac{\partial}{\partial f}-g \frac{\partial}{\partial g} . \quad Y_{5}=\frac{\partial}{\partial x}+2 f \frac{\partial}{\partial f}+g \frac{\partial}{\partial g} .
\end{gathered}
$$

The communication relations between these vector fields were given in table (a). To each s-parameter subgroup there corresponds a funnily of group invariant solutions. So, in general, it is quite impossible to determine all possible group-invariant solutions of a PDE. In order to minimize this search, it is useful to construct the optimal system of solutions. It is well known that the problem of the construction of the optimal system of solutions is equivalent to that of the construction of the optimal system of subalgebras, we will deal with the construction of the optimal system of subalgebras of $g_{s}$.

Let $G$ be a Lie group. With $g$ its Lie algebra. Each element $T \in G$ yields inner automorphism $T_{a} \rightarrow T T_{a} T^{-1}$ of the group G. Every automorphism of the group $\mathrm{G}$ induces an automorphism of $\mathrm{g}$. The set of all this automorphism is a Lie group called the adjoint group $G^{A}$. The Lie algebra of $G^{A}$ is the adjoint algebra of $g$, defined as follows. Let us have two infinitesimal generators $X, Y \in L$. The linear mapping $\operatorname{AdX}(Y): Y \rightarrow[X, Y]$ is an automorphism of $\mathrm{g}$, called the inner derivation of $\mathrm{g}$. The set of all inner derivations $\operatorname{adX}(Y)(X, Y \in)$ together with the Lie bracket $[\operatorname{AdX}, A d Y]=$ $A d[X, Y]$ is a Lie algebra $g^{A}$ called the adjoint algebra of g. Clearly $g^{A}$ is the Lie algebra of $G^{A}$. Two subalgebras in $\mathrm{g}$ are conjugate (or similar) if there is a transformation of $G^{A}$ which takes one subalgebra into the other. The collection of pairwise non-conjugate s-dimensional subalgebras is the optimal system of subalgebras of order s. The construction of one-dimensional optimal system of 
subalgebras can be carried out by using a global matrix of the adjoint transformations as suggested by L.V.Ovsiannikov (1982). The latter problem tends to determine a list (that is called an optimal system) of conjugacy inequivalent subalgebras with the property that any other subalgebra is equivalent to a unique member of the list under some element of the adjoint representation i.e. $\tilde{h} A d(g) h$ for some $g$ of a considered Lie group. Thus we will deal with the construction of the optimal system of subalgebras of $g_{5}$. The adjoint action is given by the Lie series

$$
\operatorname{Ad}\left(\exp \left(s Y_{i}\right)\right) Y_{j}=Y_{j}-s\left[Y_{i}, Y_{j}\right]+\frac{s^{2}}{2}\left[Y_{i},\left[Y_{i}, Y_{j}\right]\right]-\cdots
$$

where $\mathrm{s}$ is a parameter and $i, j=1, \ldots, 5$. The adjoint representations of $g_{5}$ is list in table (b), it consists the separate adjoint actions of each element of $g_{5}$ of all other elements.

Theorem (1.4.1): An optimal system of one-dimensional Lie subalgebras of general Burgers' equation (1.1.2) is provided by those generated by

(1) $Y^{4}=Y_{1}=\partial_{t}$,

(2) $Y^{2}=Y_{2}=\partial_{x}$,

(3) $Y^{3}=Y_{3}=\partial_{u}$,

(4) $Y^{4}=Y_{4}=t \partial_{t}+u \partial_{u}-2 f \partial_{f}-g \partial_{g}$,

(5) $Y^{5}=Y_{5}=\partial_{x}+2 f \partial_{f}+g \partial_{g}$,

(6) $Y^{6}=Y_{1}+Y_{2}=\partial_{t}+\partial_{x}$,

(7) $Y^{7}=-Y_{1}+Y_{2}=-\partial_{t}+\partial_{x}$,

(8) $Y^{8}=Y_{1}+Y_{2}=(t+1) \partial_{t}+u \partial_{u}-2 f \partial_{f}-g \partial_{g}$,

(9) $Y^{9}=-Y_{1}+Y_{4}=(t-1) \partial_{t}+u \partial_{u}-2 f \partial_{f}-g \partial_{g}$,

$$
\begin{aligned}
& Y^{10}=Y_{1}+Y_{5}=\partial_{t}+\partial_{x}+2 f \partial_{f}+g \partial_{g}, \\
& Y^{11}=-Y_{1}+Y_{5}=-\partial_{t}+\partial_{x}+2 f \partial_{f}+g \partial_{g}, \\
& Y^{12}=Y_{4}+Y_{5}=t \partial_{t}+\partial_{x}+u \partial_{u} \\
& Y^{13}=-Y_{4}+Y_{5}=-t \partial_{t}+\partial_{x}-u \partial_{u}+4 f \partial_{f}+2 g \partial_{g}, \\
& Y^{14}=Y_{1}+Y_{4}+Y_{5}=(t+1) \partial_{t}+\partial_{x}+u \partial_{u} \\
& Y^{15}=Y_{1}+Y_{4}+Y_{5}=(t+1) \partial_{t}+\partial_{x}+u \partial_{u} \\
& Y^{16}=Y_{1}-Y_{4}+Y_{5}=(t+1) \partial_{t}-u \partial_{u}+2 f \partial_{f}+g \partial_{g}, \\
& Y^{17}=-Y_{1}-Y_{4}+Y_{5}=-(1+t) \partial_{t}+\partial_{x}-u \partial_{u}+4 f \partial_{f}+2 g \partial_{g},
\end{aligned}
$$


Proof: Let $g_{4}$ is the symmetry algebra of Eq. (1.1.2) with adjoint representation determined in table (b) and

$$
Y=a_{1} Y_{1}+a_{2} Y_{2}+a_{3} Y_{3}+a_{4} Y_{4}+a_{5} Y_{5}
$$

is a nonzero vector field of $\mathrm{g}$. We will simplify as many of the coefficients $a_{i}, i=$ $1, \ldots, 5$, as possible through proper adjoint applications on $\mathrm{Y}$. We follow our aim in the below easy cases:

case1: At first, we assume that $a_{5} \neq 0$. Scaling Y if necessary, also we can assume that $a_{5}=1$ and so we get

$$
Y=a_{1} Y_{1}+a_{2} Y_{2}+a_{3} Y_{3}+a_{4} Y_{4}+Y_{5}
$$

Using the table of adjoint (table (b)). If we act on $\mathrm{Y}$ with $\operatorname{Ad}\left(\exp \left(a_{2} Y_{2}\right)\right)$, the coefficient of $Y_{2}$ can be vanished:

$$
Y^{\prime}=a_{1} Y_{1}+a_{3} Y_{3}+a_{4} Y_{4}+Y_{5}
$$

Then we apply $\operatorname{Ad}\left(\exp \left(a_{3} Y_{3}\right)\right)$ on $Y^{\prime}$ to cancel the coefficient of $Y_{3}$ :

$$
Y^{\prime \prime}=a_{1} Y_{1}+a_{4} Y_{4}+Y_{5}
$$

Case 1a: If $a_{1}, a_{4} \neq 0$ then we can make the coefficients of $Y_{1}$ and $Y_{4}$ either + 1 or -1 . Thus any one-dimensional subalgebra generated by $Y$ with $a_{3}, a_{1} \neq 0$ is equivalent to one generated by $\pm Y_{1} \pm Y_{4}+Y_{5}$ which introduce parts (14), (15), (16) and (17) of the theorem.

Case 1b: For $a_{1}=0, a_{4} \neq 0$ we can see that each one-dimensional subalgebra generated by $\mathrm{Y}$ is equivalent to one generated by $\pm Y_{4}+Y_{5}$ which introduce parts (12) and (13) of the theorem.

Case 1c: For $a_{1} \neq 0, a_{4}=0$ we can see that each one-dimensional subalgebra generated by $\mathrm{Y}$ is equivalent to one generated by $\pm Y_{1}+Y_{5}$ which introduce parts (10) and (11) of the theorem.

Case 2: The remaining one-dimensional subalgebras are spanned by vector fields of the form Y with $a_{5}=0$. 
Case 2a: If $a_{4} \neq 0$ then by scaling Y, we can assume that $a_{4}=0$. Now by the action of $\left.A d\left(\operatorname{expa}_{2} Y_{2}\right)\right)$ on Y, we can cancel the coefficient of $Y_{2}$ :

$$
\bar{Y}=a_{1} Y_{1}+a_{3} Y_{3}+Y_{4}
$$

Then by applying $A d\left(\exp \left(a_{3} Y_{3}\right)\right)$ on $\bar{Y}$ the coefficient of $Y_{3}$ can be vanished and we have

$$
\tilde{Y}^{\prime}=a_{1} Y_{1}+Y_{4}
$$

The one-dimensional subalgebra generated by $\mathrm{Y}$ is equivalent to one generated by $\pm Y_{1}+Y_{4}$ which introduce parts (8) and (9) of the theorem.

Case 2b: Let $a_{4}=0$ then $Y$ is in the form

$$
\tilde{Y}=a_{1} Y_{1}+a_{2} Y_{2}+a_{3} Y_{3}
$$

Suppose that $a_{2} \neq 0$ then if necessary we can let it equal to 1 and mew obtain

$$
\tilde{Y}^{\prime}=a_{1} Y_{1}+Y_{2}+a_{3} Y_{3}
$$

By acting $A d\left(\exp \left(a_{3} Y_{3}\right)\right)$ on $\tilde{Y}^{\prime}$, it changed to $a_{1} Y_{1}+Y_{2}$ :

Case 2b-1: Let $a_{1}$ be nonzero. In this case we can make the coefficient of $Y_{1}$ in $\tilde{Y}$ either +1 or -1 and find (6) and (7) sections of the theorem.

Case 2b-2: If $a_{1}$ is zero then $Y_{2}$ is remained. Hence this case suggests part 2).

Case 2c: Finally if in the latter case $a_{2}$ be zero, then no further simplification is possible and then $\mathrm{Y}$ is one of cases of (1.4.36). There is not any more possible case for studying and the proof is complete.

The coefficients $f, g$ of Eq. (1.1.2) depend on the variables $x, u$. Therefore, we take their optimal system's projections on the space $(x, u, f, g)$. The nonzero in $x-$ axis or $u$-axis projections of (1.4.36) are:

(1) $Z^{1}-Y^{2}=Y^{6}-Y^{7}=\partial_{x}$,

(2) $Z^{2}=Y^{3}=\partial_{u}$,

(3) $Z^{3}=Y^{4}=Y^{8}=Y^{9}=-Y^{16}=u \partial_{u}-2 f \partial_{f}-g \partial_{g}$,

(4) $Z^{4}=Y^{5}=Y^{10}=Y^{11}=\partial_{x}+2 f \partial_{f}+g \partial_{g}$, 
(5) $Z^{5}=Y^{12}=Y^{14}=Y^{15}=\partial_{x}+u \partial_{u}$,

(6) $Z^{6}=Y^{13}=Y^{17}=\partial_{x}-u \partial_{u}+4 f \partial_{f}+2 g \partial_{g}$.

Proposition (1.4.1): Let $g_{m}=\left\langle Y_{1}, \ldots, Y_{m}\right\rangle$, be an m-dimensional algebra. Denote by $Y^{i}(i=1, \ldots, r, 0<r \leq m, r \in N$ an optimal system of one-dimensional subalgebras of $g_{m}$ and by $Z^{i}(i=1, \ldots, t, 0<t \leq r, t \in N)$ the projections of $Y^{i} . i, e, Z^{i}=$ $\operatorname{pr}\left(Y^{i}\right)$. If equations

$$
f=\emptyset(x, u), \quad g=\psi(x, u)
$$

Table c: The result of the classification

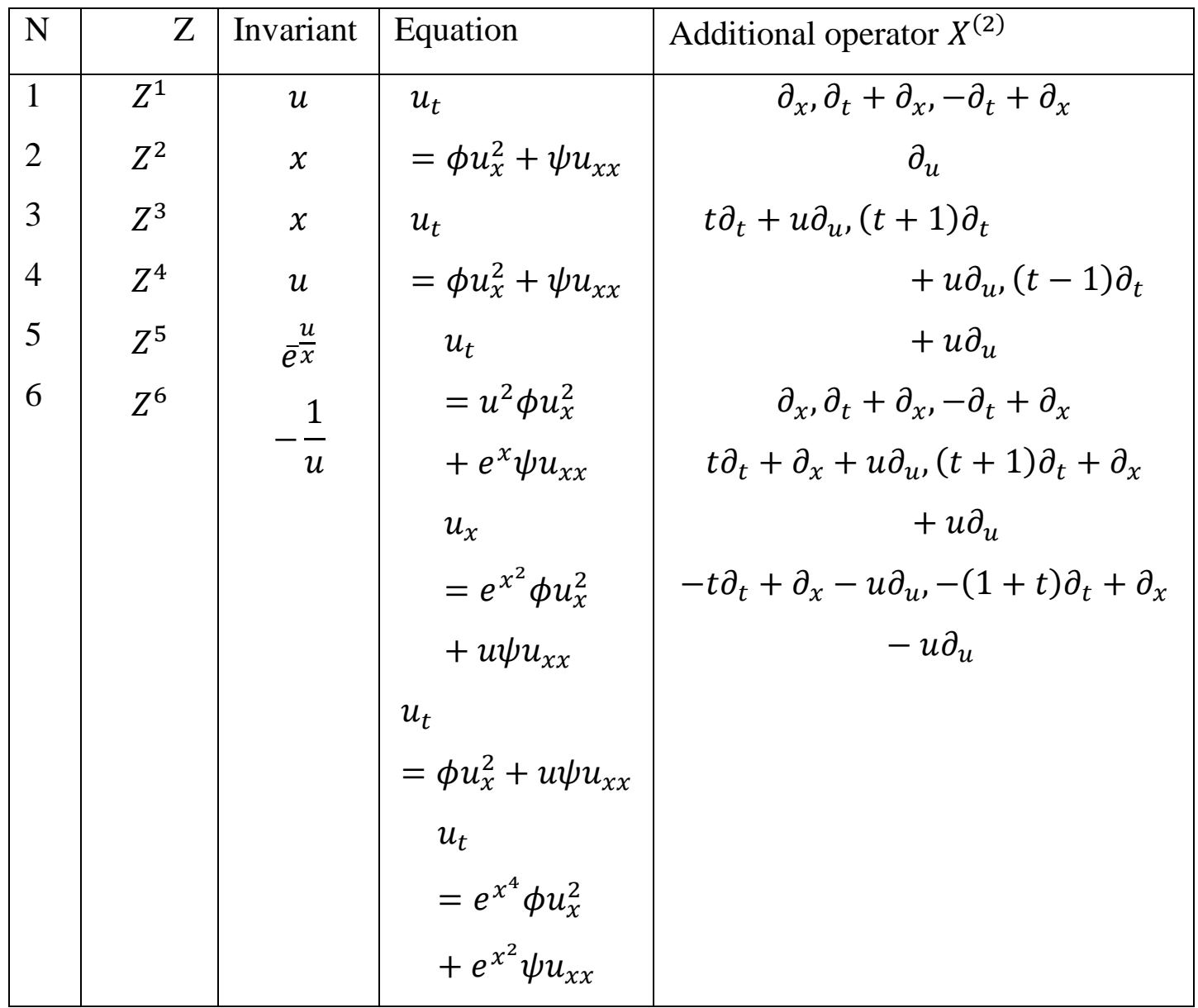

Are invariant with respect to the optimal system $Z^{i}$ then the equation

$$
u_{t}=\phi(x, u) u_{x}^{2}+\psi(x, u) u_{x x}
$$

Admits the operators $X^{i}=$ projection of $Y^{i}$ on $(t, x, u)$. 
(5) Conclusion: Finally the classical Lie method and the group classification for the class of Burgers' equation (1.1.2) and investigated the algebraic structure of the symmetry groups for this equation, is obtained. The classification is obtained by constructing an optimal system with the aid propositions (1.4.2). The result of the work is summarized in table c. of course it is also possible to obtain the corresponding reduced equations for all the cases in the classification reported in table c. we omitted these for brevity.

\section{References:}

Bluman G.W. . S. Kumei. Symmetries and Differential Equations, Springer-Verlage, World Publishing Corp., 1989.

Cantwell B.J. . Introduction to Symmetry Analysis. Cambrige University Press, 2002.

Gandarias M.L. , M. Torrisi. A. Valenti. Symmetry classification and optimal systems of a non-linear wave equation. Lnt. J. Nonlinear Mech 39 (2004)389398.

Gardner C.S. , J. M. Greene. M. . kruskal. R. M. Miura, Method for solving the Kortewegde Vries equation, Phys. Rev. Lett.19 (1967) 10951097.

Hirota R. . J. Satsuma, A variety of nonlinear network equations generated from the Bäcklund transformation for the Tota lattice, Sappl. Prog. Theor. Phys . 59(1976) 64100 .

Ibragimov N.H. . M. Tottisi. And A. Valenti. Preliminary group classification of equations $u_{x x}=f\left(x, u_{x}\right) u_{x x}+g\left(x, u_{x}\right)$.J. Math. Phys, 32. No .11:2988.2995,1991

Ibragimov N.H., M. Tottisi. And A. Valenti. Differential invariants of nonlinear equations $u_{t t}=f\left(x, u_{x}\right) u_{x x}+g\left(x, u_{x}\right)$, Communications in Nonlinear Science and and Numerical Simulation 9 (2004) 6980.

Lie S. ; Arech. For Math. 6,328 (1881)

Li Y.S. Solution and integrable system, in: Advanced Series in Nonlinear Science, Shanghai Scientific and Technological Education Publishing House. Shang Hai. 1999 (in Chinese) .

Liu H. , Jibm Li and Quanxin Zhangb. Lie symmetry analysis and exact explicit solutions for general Burgers' equation. Journal of computational and Applied Mathematics (2008). Doi:10.1016lj.cam. 2008.06.009.

Maluleke G.H. , D. P. Masom, Optimal system and group invariant solutions for the nonlinear wave equation, Communications in Nonlinear Science and Numerical Simulation 9 (2004) 93101. 
Nadjalikhah M., . Symmetries of Burgers' equation. Adv, appl. Clifford alg. DOI 10.1007\s0006-003-0000, 2008.

Nadjafikhah M., . Classification of similarity solutions for inviscid Burgers' equation. Accepted for Adv. Appl. Cliford alg., DOI 10.1007\s00006-003-0000.

Olver P.J. , Applications of Lie group to Differential Equations, in. Graduate text Maths, vol. 107, Springer, New York, 1986.

Olver P.J., Equivalence Invariants and Symmetry, Cambridge University Press, Cambridge. (1995).

Ovsiannikov L.V. . Group Analysis of Differential Equations. Academic Press.New York, 1982.

Popovych R.O. , N.M.Ivanova, New results on group classification of nonlinear diffusion-convection equations. J. Phys. A: Math. Gen, 37 (2004), 7547-7565.

Song L., and Hongging zhang, Preliminary group classification for the nonlinear wave equation $u_{t t}=f(x, u) u_{x x}+g(x, u)$, nonlinear Analysis, (2008). doi. 10.1016lj.na. 2008.07.008.

Svirshchevskii S.R. , Group classification and invariant solutions of nonlinear polyharmonic equations, Diffcr. Equ.IDiff. Uravn, 29 (1993), 1538-1547. 